\title{
Nonequilibrium dynamics in pumped Mott insulators
}

\author{
Satoshi Ejima $\odot,{ }^{*}$ Florian Lange $\odot$, and Holger Fehske $\odot$ \\ Institut für Physik, Universität Greifswald, 17489 Greifswald, Germany
}

(Received 11 October 2021; revised 6 January 2022; accepted 7 January 2022; published 9 February 2022)

\begin{abstract}
We use time-evolution techniques for (infinite) matrix product states to calculate, directly in the thermodynamic limit, the time-dependent photoemission spectra and dynamic structure factors of the half-filled Hubbard chain after pulse irradiation. These quantities exhibit clear signatures of the photoinduced phase transition from insulator to metal that occurs because of the formation of so-called $\eta$ pairs. In addition, the spin dynamic structure factor loses spectral weight in the whole momentum space, reflecting the suppression of antiferromagnetic correlations due to the buildup of $\eta$-pairing states. The numerical method demonstrated in this work can be readily applied to other one-dimensional models driven out of equilibrium by optical pumping.
\end{abstract}

DOI: 10.1103/PhysRevResearch.4.L012012

Introduction - The study of systems under optical excitation receives tremendous attention because of both the recent rapid developments of ultrafast pump lasers and the discovery of striking phenomena not observable in equilibrium, such as photoinduced superconducting-like states in high- $T_{\mathrm{c}}$ cuprates [1-3] and the alkali-doped fulleride $\mathrm{K}_{3} \mathrm{C}_{60}$ [4,5], charge-density waves in the rare-earth tritelluride $\mathrm{LaTe}_{3}$ [6], or the insulator-to-metal transition in the excitonic-insulator candidate, $\mathrm{Ta}_{2} \mathrm{NiSe}_{5}$ [7-9]. Pump-probe experiments allow to explore various physical processes at different time scales. In the prepump region the system can be still described by linear-response theory. On the other hand, the recovery process of the system in the long-time limit after a pump provides valuable information about lifetimes and interaction mechanisms of the system. Here we are interested in the rich nonequilibrium physics during or shortly after the pump. Time-dependent spectroscopic measurements, such as a timeand angle-resolved photoemission spectroscopy (TARPES) $[10,11]$, in principle enable direct comparison with theory in this time domain. It is, however, challenging to tackle these problems by numerical techniques, especially in systems with emergent photoinduced phase transitions.

Various numerical techniques have been applied to optically excited systems to study nonequilibrium dynamics, e.g., the exact-diagonalization technique [12-14], extensions of dynamical mean-field theory (DMFT) [15], and dynamical cluster approaches [16]. While ED simulations can access long times but are limited to small clusters (so far with less than 14 sites in fermionic Hubbard systems), the time-dependent DMFT is constructed directly in the thermodynamic limit but restricted by the local approximation for the

\footnotetext{
*ejima@physik.uni-greifswald.de

Published by the American Physical Society under the terms of the Creative Commons Attribution 4.0 International license. Further distribution of this work must maintain attribution to the author(s) and the published article's title, journal citation, and DOI.
}

self-energy, which may be inappropriate in low-dimensional systems with inherent nonlocal spatial correlations. In one dimension, methods based on matrix product states (MPSs), such as the density-matrix renormalization group (DMRG) $[17,18]$ and its time-dependent version $[19,20]$, enable us to simulate both static and dynamic properties with high accuracy even for large systems. Results for nonequilibrium dynamics are still rare, however. The time evolution of the spectral functions after a quantum quench has been computed in the extended Hubbard chain at half filling for finite systems [21-25]. Very recently, photodoped Mott insulators have been studied by an infinite time-evolving block decimation (iTEBD) approach [26,27] with the help of a generalized Gibbs ensemble to obtain an appropriate effective model description at equilibrium [28].

In this paper, we propose a direct numerical scheme for the computation of nonequilibrium dynamic response functions, which can be used for general (quasi-)one-dimensional (1D) systems. We apply this technique to the optically excited half-filled Hubbard chain, in which a photoinduced $\eta$-pairing state appears [29]. In the time-resolved photoemission spectra (PES), an extra band becomes visible above the Fermi energy after pulse irradiation, indicating the insulator-to-metal phase transition. Further evidence for this transition can be found in the charge dynamic structure factor (DSF), where a gapless band appears.

Nonequilibrium dynamics-To explore the system's dynamics at nonequilibrium, time-dependent spectral functions of the form $[13,14,30]$

$$
\begin{aligned}
I(k, \omega ; t)= & \sum_{r} e^{-i k r} \int_{-\infty}^{\infty} \int_{-\infty}^{\infty} d \tau_{1} d \tau_{2} f\left(\tau_{1}, \tau_{2} ; \omega\right) \\
& \times C\left(r, \tau_{1}, \tau_{2} ; t\right)
\end{aligned}
$$

are of interest. Here $C\left(r, \tau_{1}, \tau_{2} ; t\right)=\langle\phi(t)| \hat{O}_{j+r}^{\dagger}$ $\left(\tau_{1} ; t\right) \hat{O}_{j}\left(\tau_{2} ; t\right)|\phi(t)\rangle$ is the nonequilibrium two-point correlator at times $\tau_{1}$ and $\tau_{2}$ defined relative to time $t$ for a local operator $\hat{O}_{j}$. The prefactor is given by $f\left(\tau_{1}, \tau_{2} ; \omega\right)=$ $e^{i \omega\left(\tau_{1}-\tau_{2}\right)} g\left(\tau_{1}\right) g\left(\tau_{2}\right) \quad$ with $\quad g(\tau)=\exp \left[-\tau^{2} / 2 \sigma_{\mathrm{pr}}^{2}\right] / \sqrt{2 \pi} \sigma_{\mathrm{pr}}$ 
describing the shape of a probe pulse, e.g., in a timedependent photoemission spectroscopy experiment.

We calculate the correlator $C\left(r, \tau_{1}, \tau_{2} ; t\right)$ numerically by simulating states $\hat{O}_{j}(\tau ; t)|\phi(t)\rangle=\hat{U}^{\dagger}(t+\tau, t) \hat{O}_{j} \hat{U}(t+$ $\tau, t)|\phi(t)\rangle \equiv|\bar{\phi}(\tau ; t)\rangle$ within an (i)MPS representation. Here $\hat{U}(t+\tau, t) \equiv \mathcal{T} \exp \left[-i \int_{t}^{t+\tau} d t^{\prime} \hat{H}\left(t^{\prime}\right)\right]$ is the unitary timeevolution operator of the system with the (reverse) timeordering operator $\mathcal{T}$ for $\tau>0(\tau<0)$. To this end, we first prepare the iMPS approximation $\left|\psi_{0}\right\rangle$ of the ground state of the unperturbed Hamiltonian $\hat{H}$ by infinite DMRG [31]. We then utilize the iTEBD technique to carry out a time evolution according to the time-dependent Hamiltonian $\hat{H}(t)$, which includes an electric-field $A(t)$, in order to obtain an iMPS representation of the photoinduced state $|\psi(t)\rangle$. Using the iMPS description reduces the numerical cost, since the size of the unit cell $N_{\text {uc }}$ is usually much smaller than the system sizes needed in simulations with open boundary conditions. Applying the operator $\hat{O}_{j}$ lifts the translation invariance of the state, however, so we need to switch to an MPS representation with infinite boundary conditions (IBCs) for the rest of the simulation. More specifically, "Method I" described in Ref. [32] is necessary because the photoexcited state is not an eigenstate of the Hamiltonian that determines the time evolution. Having calculated states $|\bar{\phi}(\tau ; t)\rangle$ for times $\tau_{1}$ and $\tau_{2}$, the two-point correlators $C\left(r, \tau_{1}, \tau_{2} ; t\right)$ in Eq. (1) can be evaluated by shifting $\left|\bar{\phi}\left(\tau_{1} ; t\right)\right\rangle$ and $\left|\bar{\phi}\left(\tau_{2} ; t\right)\right\rangle$ relative to each other $[33,34]$. In this way, the number of MPSs $|\bar{\phi}(\tau ; t)\rangle$ required for some fixed time $\tau$ is drastically reduced. Namely, we only need a number of MPSs proportional to $N_{\text {uc }}$ instead of $L$ as in a similar approach with open boundary conditions. Further technical details are given in the Supplemental Material [35].

Model-Our target system is the 1D half-filled Hubbard model with nearest-neighbor hopping $t_{\mathrm{h}}$ and on-site Coulomb repulsion $U>0$,

$$
\begin{aligned}
\hat{H}= & -t_{\mathrm{h}} \sum_{j, \sigma}\left(\hat{c}_{j, \sigma}^{\dagger} \hat{c}_{j+1, \sigma}+\text { H.c. }\right) \\
& +U \sum_{j}\left(\hat{n}_{j, \uparrow}-1 / 2\right)\left(\hat{n}_{j, \downarrow}-1 / 2\right),
\end{aligned}
$$
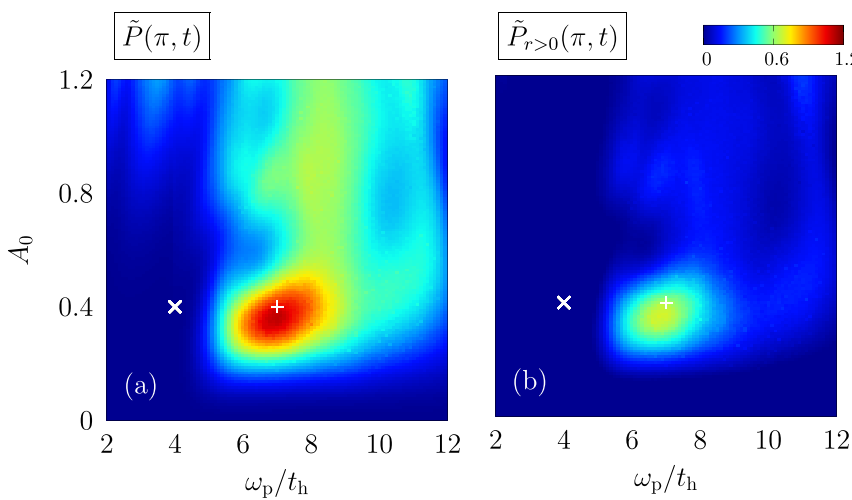

FIG. 1. Contour plots of $\tilde{P}\left(\pi, t=15 t_{\mathrm{h}}^{-1}\right)$ (a) and $\tilde{P}_{r>0}(\pi, t=$ $\left.15 t_{\mathrm{h}}^{-1}\right)(\mathrm{b})$ in the $\omega_{\mathrm{p}}-A_{0}$ plane for an infinite Hubbard chain with $U / t_{\mathrm{h}}=8$ at half filling. The pump pulse has width $\sigma_{\mathrm{p}}=2$ and is centered at time $t_{0} \cdot t_{\mathrm{h}}=10$. where $\hat{c}_{j, \sigma}^{\dagger}\left(\hat{c}_{j, \sigma}\right)$ creates (annihilates) a fermion with spin projection $\sigma(=\uparrow, \downarrow)$ at lattice site $j$, and $\hat{n}_{j, \sigma}=$ $\hat{c}_{j, \sigma}^{\dagger} \hat{c}_{j, \sigma}$. The ground state for $U>0$ is a Mott insulator with a finite charge gap $\Delta$. As Yang demonstrated in a seminal paper [36], exact eigenstates of the Hubbard model can be constructed by means of the so-called $\eta$
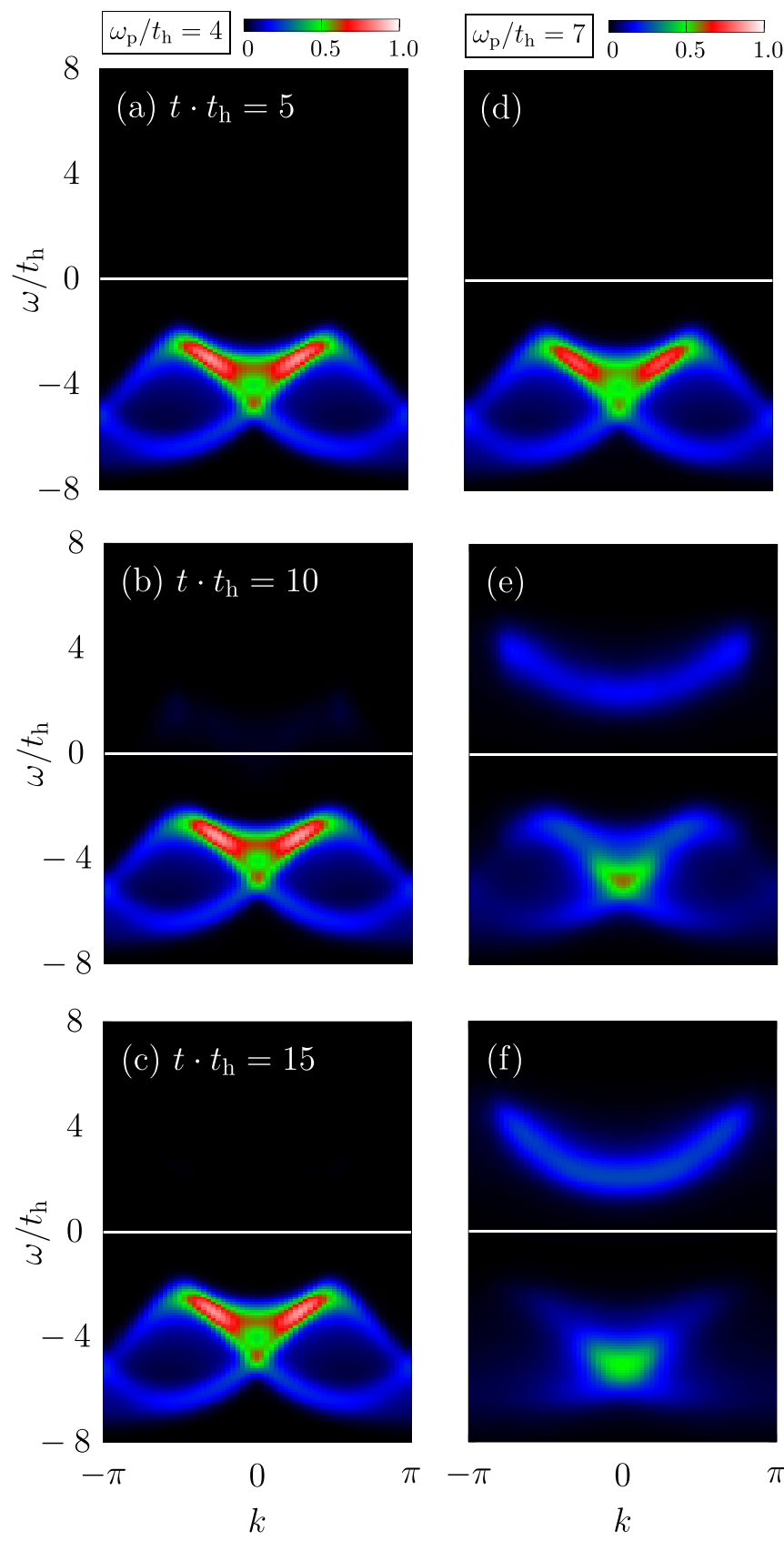

FIG. 2. Snapshots of the photoemission spectra $A^{-}(k, \omega ; t)$ for the $\eta$-pairing non-dominant (left panels with $\left.\omega_{\mathrm{p}} / t_{\mathrm{h}}=4\right)$ and dominant (right panels with $\omega_{\mathrm{p}} / t_{\mathrm{h}}=7$ ) states during the pump at $t \cdot t_{\mathrm{h}}=5$ [(a) and (d)], 10 [(b) and (e)], and 15 [(c) and (f)]. The data are obtained by the (i)TEBD technique with IBCs at $U / t_{\mathrm{h}}=8$, where the pump is parametrized by $A_{0}=4$ and $\sigma_{\mathrm{p}}=2$ at $t_{0} \cdot t_{\mathrm{h}}=10$. 
operators,

$$
\begin{gathered}
\hat{\eta}^{+}=\sum_{j}(-1)^{j} \hat{\Delta}_{j}^{\dagger} \equiv \sum_{j} \hat{\eta}_{j}^{+}, \quad \hat{\eta}^{-}=\left(\hat{\eta}^{+}\right)^{\dagger}, \\
\hat{\eta}^{z}=\frac{1}{2} \sum_{j}\left(\hat{n}_{j, \uparrow}+\hat{n}_{j, \downarrow}-1\right) \equiv \sum_{j} \hat{\eta}_{j}^{z},
\end{gathered}
$$

which obey the SU(2) commutation relations; $\hat{\Delta}_{j}^{\dagger}=\hat{c}_{j, \downarrow}^{\dagger} \hat{c}_{j, \uparrow}^{\dagger}$ is the singlet pair-creation operator.

The Hubbard Hamiltonian (2) commutes with the operator $\hat{\eta}^{2}=\frac{1}{2}\left(\hat{\eta}^{+} \hat{\eta}^{-}+\hat{\eta}^{-} \hat{\eta}^{+}\right)+\left(\hat{\eta}^{z}\right)^{2}$, so that $\left\langle\eta^{2}\right\rangle$ is a conserved quantity in the absence of perturbations. Eigenstates with a finite value of $\left\langle\hat{\eta}^{2}\right\rangle$ have long-ranged pairing correlations $\left\langle\hat{\eta}_{j}^{+} \hat{\eta}_{\ell}^{-}\right\rangle$[36]. While these $\eta$-pairing states cannot be ground states, it has been recently recognized that pulse irradiation can induce $\eta$-pairing in Mott insulators [29]. Here we study such photoinduced $\eta$-pairing states with the time-evolution technique outlined above.

We introduce the external time-dependent electricfield $A(t)$ via a Peierls phase [37] as $t_{\mathrm{h}} \hat{c}_{j, \sigma}^{\dagger} \hat{c}_{j+1, \sigma} \rightarrow$ $t_{\mathrm{h}} e^{\mathrm{i} A(t)} \hat{c}_{j, \sigma}^{\dagger} \hat{c}_{j+1, \sigma}$, where

$$
A(t)=A_{0} e^{-\left(t-t_{0}\right)^{2} /\left(2 \sigma_{\mathrm{p}}^{2}\right)} \cos \left[\omega_{\mathrm{p}}\left(t-t_{0}\right)\right] .
$$

This describes a pump pulse with amplitude $A_{0}$, frequency $\omega_{\mathrm{p}}$, and width $\sigma_{\mathrm{p}}$ centered at time $t_{0}(>0)$. As a result, the Hamiltonian becomes time dependent, $\hat{H} \rightarrow \hat{H}(t)$, and the system being initially in the ground state is driven out of equilibrium, $|\psi(0)\rangle \rightarrow|\psi(t)\rangle$.

The $\eta$-pairing state can be detected by evaluating the time evolution of the pair-correlation function

$$
\left.P(r, t)=\frac{1}{L} \sum_{j}\langle\psi(t)| \hat{\Delta}_{j+r}^{\dagger} \hat{\Delta}_{j}+\text { H.c. }\right)|\psi(t)\rangle
$$

and its Fourier transform $\tilde{P}(q, t)=\sum_{r} e^{i q r} P(r, t)$. As demonstrated in Refs. [29,38] for small clusters and in Ref. [39] in the thermodynamic limit, $\tilde{P}(\pi, t)$ is enhanced after pulse irradiation, which implies the formation of an $\eta$-pairing state. The optimal parameter set for inducing $\eta$-pairing thus can be determined examining the $A_{0}$ and $\omega_{\mathrm{p}}$ dependences of $\tilde{P}(\pi, t)$ with the iTEBD technique [39].

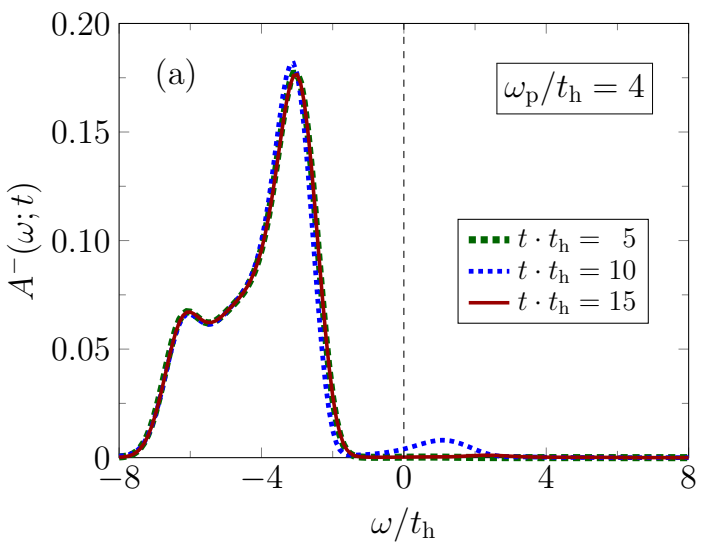

Figure 1(a) shows the contour plot of $\tilde{P}(\pi, t)$ after pulse irradiation $\left(t \cdot t_{\mathrm{h}}=15\right)$. Obviously, there is a maximum around $A_{0} \approx 0.4$ and $\omega_{\mathrm{p}} / t_{\mathrm{h}} \approx 7.0$ marked by “ + ." In the following, we analyze the nonequilibrium spectral functions for this parameter set. To demonstrate that the nonlocal part of the pairing correlations is dominant for these optimal parameters, we also extract the contour plot of the modified structure factor $\tilde{P}_{r>0}(q, t)=\sum_{r>0} e^{i q r} P(r, t)$, in which the contribution of the double occupancy $n_{\mathrm{d}}(t)=(1 / L) \sum_{j}\left\langle\psi(t)\left|\hat{n}_{j, \uparrow} \hat{n}_{j, \downarrow}\right| \psi(t)\right\rangle$ is excluded [Fig. 1(b)]. Clearly the spectral intensity of $\tilde{P}_{r>0}(\pi, t)$ is only appreciable in the vicinity of + . We also explore the nonequilibrium dynamics away from the optimal parameter set at $A_{0}=0.4$ and $\omega_{\mathrm{p}}=4.0$ (denoted as $\times$ in Fig. 1) to elucidate the difference between $\eta$-pair-correlation dominant and nondominant regions.

PES-Since the 1D half-filled Hubbard model has an insulating ground state for any $U>0$, there exists a Mott gap $(\Delta \sim U)$ between lower and upper Hubbard bands in the single-particle spectral function $A(k, \omega)=A^{+}(k, \omega)+$ $A^{-}(k, \omega)$, where $A^{-}(k, \omega)$ and $A^{+}(k, \omega)$ denote the PES and inverse PES, respectively. In the superconducting $\eta$-pairing state after pulse irradiation, however, the Mott gap is melted, which should also be captured in the time-dependent singleparticle spectral function $A(k, \omega ; t)$. Because of particle-hole symmetry, $A^{+}(k, \omega ; t)$ and $A^{-}(k, \omega ; t)$ contain the same information. We focus on $A^{-}(k, \omega ; t)$, which is obtained by setting $\hat{O}_{j}=\hat{c}_{j, \sigma}$ in Eq. (1).

Figure 2 displays our (i)TEBD results for $A^{-}(k, \omega ; t)$, using the pump parameter sets ' $X$ ' and ' + ' of Fig. 1, which correspond to angular frequencies $\omega_{\mathrm{p}} / t_{\mathrm{h}}=4$ and $\omega_{\mathrm{p}} / t_{\mathrm{h}}=7$, respectively. For $\omega_{\mathrm{p}} / t_{\mathrm{h}}=4$, the time-dependent spectral function is very similar to the equilibrium spectral function at $T=0$ (see, e.g., Ref [34]), i.e., it is only slightly changed by the pulse irradiation. In contrast, when the parameters optimized to induce $\eta$-pairing are used $\left(\omega_{\mathrm{p}} / t_{\mathrm{h}}=7\right)$, an extra dispersion above the Fermi energy $\left(\omega>E_{\mathrm{F}}\right)$ appears during the pump and persists afterwards [Figs. 2(e)-2(f)].

Evaluating the integrated density of states

$$
A^{-}(\omega ; t)=\frac{1}{L} \sum_{k} A^{-}(k, \omega ; t),
$$

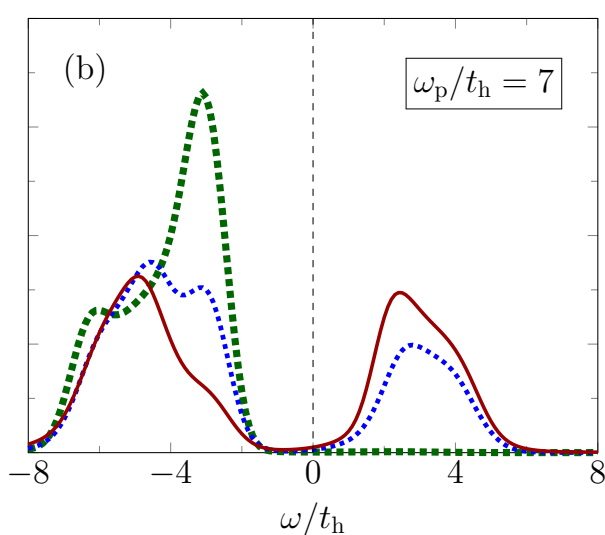

FIG. 3. The transient integrated density of states $A^{-}(\omega ; t)$ from Eq. (7) for $U / t_{\mathrm{h}}=8$ with $\omega_{\mathrm{p}} / t_{\mathrm{h}}=4$ (a) and 7 (b). The pump is parametrized as in Fig. 2. 
we see more clearly how the spectral weight is shifted from $\omega<E_{\mathrm{F}}$ to $\omega>E_{\mathrm{F}}$ by the photoinduced $\eta$-pairing. Figure 3(a) shows $A^{-}(\omega ; t)$ for $\omega_{\mathrm{p}} / t_{\mathrm{h}}=4$. Although a small shift of the spectral weight to $\omega>E_{\mathrm{F}}$ is observed at $t \approx t_{0}$, it becomes negligible after the pulse $\left(t \cdot t_{\mathrm{h}} \gtrsim 15\right)$. On the other hand, the spectral weight for $\omega>E_{\mathrm{F}}$ increases distinctly over time in the $\omega_{\mathrm{p}} / t_{\mathrm{h}}=7$ case [Fig. 3(b)], indicating a photoinduced phase transition from a Mott insulator to a metallic $\eta$-pairing state. This photoinduced insulator-to-metal transition should be observed in TARPES, when the pure Hubbard model is realized experimentally, e.g., in optical lattices (although this would require further developments of ARPES techniques [40]).

$D S F-W e$ now analyze the spin and charge DSFs, $S_{\mathrm{s}}(q, \omega ; t)$ and $S_{\mathrm{c}}(q, \omega ; t)$, which are obtained by setting $\hat{O}_{j}=$ $\hat{S}_{j}^{z}$ and $\hat{O}_{j}=\hat{n}_{j \uparrow}+\hat{n}_{j \downarrow}-1$ in Eq. (1), respectively.

Let us first recall the character of the DSF at zero temperature. For any $U>0$, the spin DSF consists of a two-spinon continuum with an excitation gap that closes at momenta $q=0$ and $\pi$, while the charge DSF is gapful, reflecting the Mott gap for holon excitations (see also the numerical results with IBCs in Ref. [35]).

Figures 4(a)-4(c) give the nonequilibrium spin DSF $S_{\mathrm{s}}(q, \omega ; t)$ in the half-filled Hubbard model with $U / t_{\mathrm{h}}=8$ using the optimal parameter set (+ from Fig. 1). In Fig. 4(a) $S_{\mathrm{s}}(q, \omega ; t)$ is quite similar to $S_{\mathrm{s}}(q, \omega)$ at equilibrium, showing the gap closing at the momenta $q=0$ and $\pi$. During and after pulse irradiation [Figs. 4(b) and 4(c)], the spectral weight is reduced in the whole momentum space, reflecting the suppression of antiferromagnetic correlations in the photoinduced $\eta$-pairing state [29].

More drastic changes should be expected in the charge DSF, since $S_{\mathrm{c}}(q, \omega)$ can be written with the $\eta$ operators in Eqs. (3) and (4) as discussed in Refs. [35,41]. This is in accordance with the numerical results shown in Figs. 4(d) and $4(\mathrm{e})$. Before the pump pulse [Fig. 4(d)], $S_{\mathrm{c}}(q, \omega ; t)$ is nearly indistinguishable from the equilibrium $\operatorname{DSF}_{\mathrm{c}}(q, \omega)$ at $T=0$, with the Mott gap $\left(\Delta_{\mathrm{c}} \approx 4.68\right.$ for $\left.U / t_{\mathrm{h}}=8\right)$ visible at $q=0$. During the pump $\left(t=t_{0}\right)$ an extra band appears and closes the gap [Fig. 4(e)]. Most significantly, the spectral weight now concentrates at $q \approx 0.55$ and $\omega \approx 0$, which is confirmed more clearly in the energy-integrated charge structure factor $S_{\mathrm{c}}(q ; t)=\int_{-\infty}^{\infty} d \omega S_{\mathrm{c}}(q, \omega ; t)$, as shown in Fig. 5. Note that $S_{\mathrm{c}}(q ; t)$ can also be obtained from the Fourier transform of the two-point correlation functions $\left\langle\psi(t)\left|\hat{\eta}_{j+r}^{z} \hat{\eta}_{j}^{z}\right| \psi(t)\right\rangle$ using iTEBD in the iMPS representation:

$$
\begin{aligned}
S_{\mathrm{c}}(q ; t) & =4 \int_{-\infty}^{\infty} d \tau \frac{e^{-\tau^{2} / \sigma_{\mathrm{pr}}^{2}}}{\sigma_{\mathrm{pr}}^{2}} \sum_{r} e^{-i q r}\left\langle\psi(t)\left|\hat{\eta}_{j+r}^{z} \hat{\eta}_{j}^{z}\right| \psi(t)\right\rangle \\
& =\frac{4 \sqrt{\pi}}{\sigma_{\mathrm{pr}}} \sum_{r} e^{-i q r}\left\langle\psi(t)\left|\hat{\eta}_{j+r}^{z} \hat{\eta}_{j}^{z}\right| \psi(t)\right\rangle .
\end{aligned}
$$

This peak structure might be taken as an indication for superfluidity of the $\eta$-pairing state. It would be desirable to extract an order parameter with the help of field-theoretical analysis analogous to the Tomonaga-Luttinger liquid parameter in the 1D Bose-Hubbard model, which characterizes the superfluid phase; see, e.g., Ref. [42]. Note that in Ref. [22],
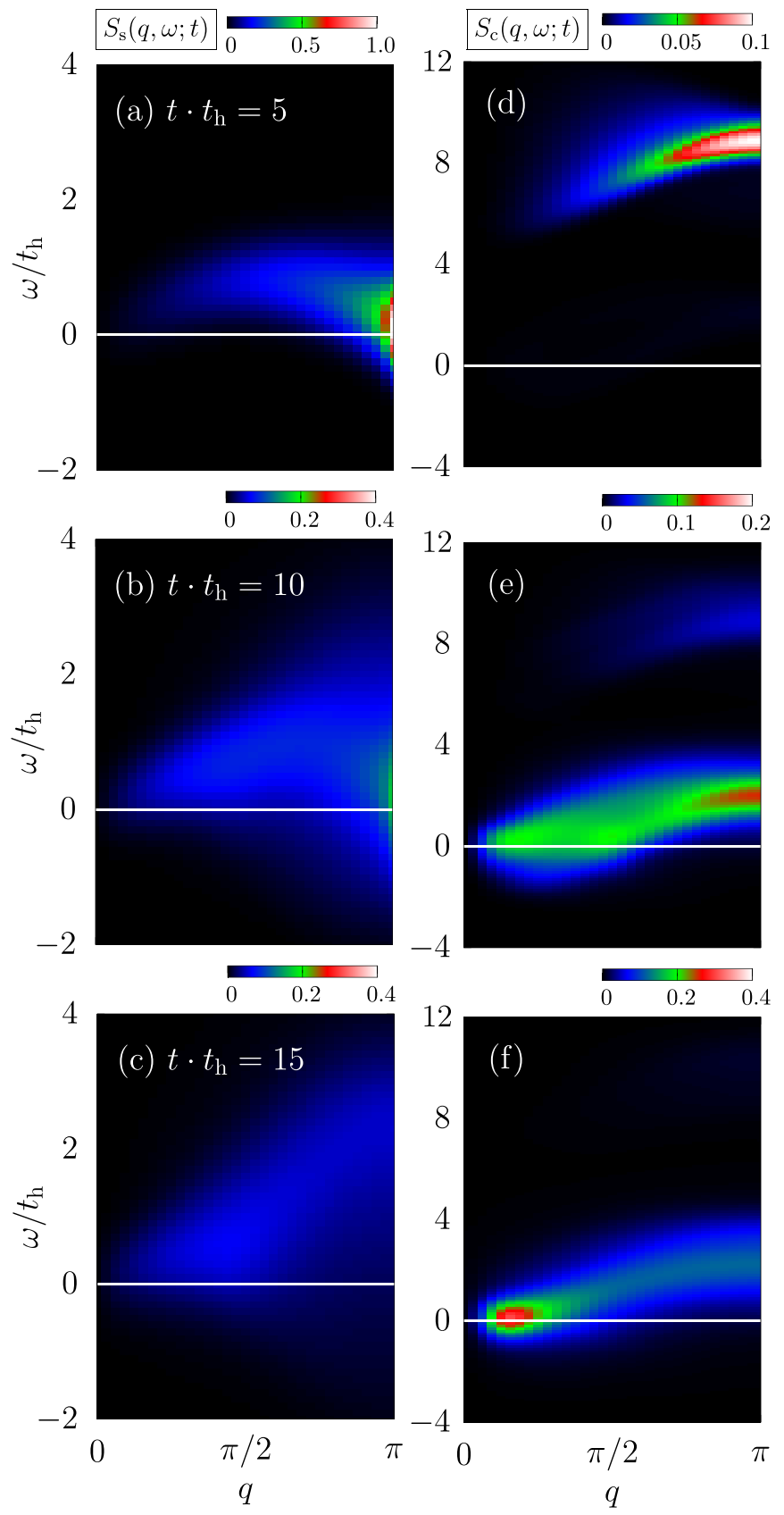

FIG. 4. Snapshots of the dynamic structure factors for spin $\left[S_{\mathrm{s}}(q, \omega ; t)\right]$ and charge $\left[S_{\mathrm{c}}(q, \omega ; t)\right]$ during the pump at $t \cdot t_{\mathrm{h}}=5$ [(a) and (d)], 10 [(b) and (e)], and 15 [(c) and (f)]. The data are obtained by the time-evolution technique with IBCs, where the pump is parametrized by $A_{0}=0.4$ and $\omega_{\mathrm{p}} / t_{\mathrm{h}}=7$ ('+' symbol in Fig. 1).

a related two-particle spectral function $\left[\hat{O}_{j}=\hat{\Delta}_{j}\right]$ was studied to show a nonequilibrium transition to a superconducting phase.

Conclusions-We have demonstrated how the spectral functions of (quasi-)1D systems at nonequilibrium can be simulated directly in the thermodynamic limit by using the time-dependent DMRG technique with IBCs. We have applied this technique to the optically excited Hubbard chain at half filling and observed that so-called $\eta$-pairing states appear after pulse irradiation. Tuning the pump pulse to maximize 


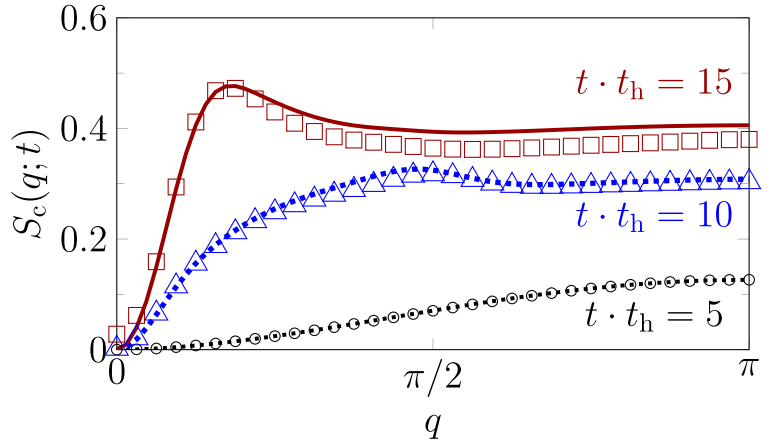

FIG. 5. The time-dependent charge structure factor $S_{\mathrm{c}}(q ; t)$ for $\omega_{\mathrm{p}} / t_{\mathrm{h}}=7$ obtained from the energy-integrated $S_{\mathrm{c}}(q, \omega ; t)$ (symbols) and the Fourier transform of $\left\langle\psi(t)\left|\hat{\eta}_{j+r}^{z} \hat{\eta}_{j}^{z}\right| \psi(t)\right\rangle$ [Eq. (8)] (lines), where the pump is parametrized as in Fig. 4.

$\eta$-pairing, we have found clear evidence for a photoinduced metal-insulator transition in both the time-dependent PES and DSFs.
Some care should be taken when using the spectral function $I(k, \omega ; t)$ from Eq. (1) to interpret TARPES experiments, since its derivation relies on the assumption that the pump and probe pulses do not overlap. For overlapping pulses, gauge invariance may be violated [43]. Importantly, however, this does not affect the results for long times $\left(t \cdot t_{\mathrm{h}}=15\right)$, which are the most relevant to our conclusions. Moreover, there is no problem related to gauge invariance for the integrated density of states shown in Fig. 3, which is thus also valid.

The proposed numerical scheme opens a new venue for exploring nonequilibrium dynamics with high resolution and controllable accuracy. It would be of specific interest to try to numerically reproduce the experimental results of timedependent ARPES of $\mathrm{Ta}_{2} \mathrm{NiSe}_{5}$ [7-9], which is a strong candidate for excitonic insulators and exhibits the characteristic flat-band behavior in ARPES experiments [44,45] within a narrow region in momentum space [34].

Acknowledgments - The iTEBD simulations were performed using the ITensor library [46]. S.E. and F.L. were supported by Deutsche Forschungsgemeinschaft through project EJ 7/2-1 and FE 398/8-1, respectively.
[1] D. Fausti, R. I. Tobey, N. Dean, S. Kaiser, A. Dienst, M. C. Hoffmann, S. Pyon, T. Takayama, H. Takagi, and A. Cavalleri, Light-induced superconductivity in a stripe-ordered cuprate, Science 331, 189 (2011).

[2] W. Hu, S. Kaiser, D. Nicoletti, C. R. Hunt, I. Gierz, M. C. Hoffmann, M. L. Tacon, T. Loew, B. Keimer, and A. Cavalleri, Optically enhanced coherent transport in $\mathrm{YBa}_{2} \mathrm{Cu}_{3} \mathrm{O}_{6.5}$ by ultrafast redistribution of interlayer coupling, Nat. Mater. 13, 705 (2014).

[3] S. Kaiser, C. R. Hunt, D. Nicoletti, W. Hu, I. Gierz, H. Y. Liu, M. Le Tacon, T. Loew, D. Haug, B. Keimer, and A. Cavalleri, Optically induced coherent transport far above $T_{c}$ in underdoped $\mathrm{YBa}_{2} \mathrm{Cu}_{3} \mathrm{O}_{6+\delta}$, Phys. Rev. B 89, 184516 (2014).

[4] M. Mitrano, A. Cantaluppi, D. Nicoletti, S. Kaiser, A. Perucchi, S. Lupi, P. Di Pietro, D. Pontiroli, M. Riccò, S. R. Clark, D. Jaksch, and A. Cavalleri, Possible light-induced superconductivity in $\mathrm{K}_{3} \mathrm{C}_{60}$ at high temperature, Nature (London) 530, 461 (2016).

[5] M. Budden, T. Gebert, M. Buzzi, G. Jotzu, E. Wang, T. Matsuyama, G. Meier, Y. Laplace, D. Pontiroli, M. Riccò, F. Schlawin, D. Jaksch, and A. Cavalleri, Evidence for metastable photo-induced superconductivity in $\mathrm{K}_{3} \mathrm{C}_{60}$, Nat. Phys. 17, 611 (2021).

[6] A. Kogar, A. Zong, P. E. Dolgirev, X. Shen, J. Straquadine, Y.-Q. Bie, X. Wang, T. Rohwer, I.-C. Tung, Y. Yang, R. Li, J. Yang, S. Weathersby, S. Park, M. E. Kozina, E. J. Sie, H. Wen, P. Jarillo-Herrero, I. R. Fisher, X. Wang, and N. Gedik, Light-induced charge density wave in $\mathrm{LaTe}_{3}$, Nat. Phys. 16, 159 (2020).

[7] S. Mor, M. Herzog, D. Golež, P. Werner, M. Eckstein, N. Katayama, M. Nohara, H. Takagi, T. Mizokawa, C. Monney, and J. Stähler, Ultrafast Electronic Band Gap Control in an Excitonic Insulator, Phys. Rev. Lett. 119, 086401 (2017).

[8] K. Okazaki, Y. Ogawa, T. Suzuki, T. Yamamoto, T. Someya, S. Michimae, M. Watanabe, Y. Lu, M. Nohara, H. Takagi, N. Katayama, H. Sawa, M. Fujisawa, T. Kanai, N. Ishii, J. Itatani,
T. Mizokawa, and S. Shin, Photo-induced semimetallic states realised in electron-hole coupled insulators, Nat. Commun. 9, 4322 (2018).

[9] T. Tang, H. Wang, S. Duan, Y. Yang, C. Huang, Y. Guo, D. Qian, and W. Zhang, Non-Coulomb strong electron-hole binding in $\mathrm{Ta}_{2} \mathrm{NiSe}_{5}$ revealed by time- and angle-resolved photoemission spectroscopy, Phys. Rev. B 101, 235148 (2020).

[10] X. Zhou, S. He, G. Liu, L. Zhao, L. Yu, and W. Zhang, New developments in laser-based photoemission spectroscopy and its scientific applications: A key issues review, Rep. Prog. Phys. 81, 062101 (2018).

[11] B. Lv, T. Qian, and H. Ding, Angle-resolved photoemission spectroscopy and its application to topological materials, Nat. Rev. Phys. 1, 609 (2019).

[12] T. J. Park and J. C. Light, Unitary quantum time evolution by iterative Lanczos reduction, J. Chem. Phys. 85, 5870 (1986).

[13] Y. Wang, M. Claassen, B. Moritz, and T. P. Devereaux, Producing coherent excitations in pumped mott antiferromagnetic insulators, Phys. Rev. B 96, 235142 (2017).

[14] J. Okamoto, Time-dependent spectral properties of a photoexcited one-dimensional ionic Hubbard model: an exact diagonalization study, New J. Phys. 21, 123040 (2019).

[15] H. Aoki, N. Tsuji, M. Eckstein, M. Kollar, T. Oka, and P. Werner, Nonequilibrium dynamical mean-field theory and its applications, Rev. Mod. Phys. 86, 779 (2014).

[16] N. Bittner, D. Golež, M. Eckstein, and P. Werner, Photoenhanced excitonic correlations in a Mott insulator with nonlocal interactions, Phys. Rev. B 101, 085127 (2020).

[17] S. R. White, Density Matrix Formulation for Quantum Renormalization Groups, Phys. Rev. Lett. 69, 2863 (1992).

[18] U. Schollwöck, The density-matrix renormalization group in the age of matrix product states, Ann. Phys. 326, 96 (2011).

[19] S. R. White and A. E. Feiguin, Real-Time Evolution Using the Density Matrix Renormalization Group, Phys. Rev. Lett. 93, 076401 (2004). 
[20] A. J. Daley, C. Kollath, U. Schollwöck, and G. Vidal, Timedependent density-matrix renormalization-group using adaptive effective Hilbert spaces, J. Stat. Mech. Theory Exp. (2004) P04005.

[21] K. Zawadzki and A. E. Feiguin, Time- and momentum-resolved tunneling spectroscopy of pump-driven nonthermal excitations in Mott insulators, Phys. Rev. B 100, 195124 (2019).

[22] S. Paeckel, B. Fauseweh, A. Osterkorn, T. Köhler, D. Manske, and S. R. Manmana, Detecting superconductivity out of equilibrium, Phys. Rev. B 101, 180507(R) (2020).

[23] K. Zawadzki, L. Yang, and A. E. Feiguin, Time-dependent approach to inelastic scattering spectroscopies in and away from equilibrium: Beyond perturbation theory, Phys. Rev. B 102, 235141 (2020).

[24] J. Rincón and A. E. Feiguin, Nonequilibrium optical response of a one-dimensional Mott insulator, Phys. Rev. B 104, 085122 (2021).

[25] C. Meyer and S. R. Manmana, Formation of spinful dark excitons in Hubbard systems with magnetic superstructures, arXiv:2109.07037.

[26] G. Vidal, Efficient Classical Simulation of Slightly Entangled Quantum Computations, Phys. Rev. Lett. 91, 147902 (2003).

[27] G. Vidal, Classical Simulation of Infinite-Size Quantum Lattice Systems in One Spatial Dimension, Phys. Rev. Lett. 98, 070201 (2007).

[28] Y. Murakami, S. Takayoshi, T. Kaneko, Z. Sun, D. Golež, A. J. Millis, and P. Werner, Exploring nonequilibrium phases of photo-doped Mott insulators with generalized Gibbs ensembles, Commun. Phys. 5, 23 (2022).

[29] T. Kaneko, T. Shirakawa, S. Sorella, and S. Yunoki, Photoinduced $\eta$ Pairing in the Hubbard Model, Phys. Rev. Lett. 122, 077002 (2019).

[30] J. K. Freericks, H. R. Krishnamurthy, and T. Pruschke, Theoretical Description of Time-Resolved Photoemission Spectroscopy: Application to Pump-Probe Experiments, Phys. Rev. Lett. 102, 136401 (2009).

[31] I. P. McCulloch, Infinite size density matrix renormalization group, revisited, arXiv:0804.2509.

[32] V. Zauner, M. Ganahl, H. G. Evertz, and T. Nishino, Time evolution within a comoving window: scaling of signal fronts and magnetization plateaus after a local quench in quantum spin chains, J. Phys.: Condens. Matter 27, 425602 (2015).

[33] F. Lange, S. Ejima, and H. Fehske, Finite-temperature dynamic structure factor of the spin-1 XXZ chain with single-ion anisotropy, Phys. Rev. B 97, 060403(R) (2018).

[34] S. Ejima, F. Lange, and H. Fehske, Finite-temperature photoemission in the extended Falicov-Kimball model: a case study for $\mathrm{Ta}_{2} \mathrm{NiSe}_{5}$, SciPost Phys. 10, 077 (2021).

[35] See Supplemental Material at http://link.aps.org/supplemental/ 10.1103/PhysRevResearch.4.L012012 for details, which includes Refs. $[47,48]$.

[36] C. N. Yang, $\eta$ Pairing and Off-Diagonal Long-Range Order in a Hubbard Model, Phys. Rev. Lett. 63, 2144 (1989).

[37] R. Peierls, Zur theorie des diamagnetismus von leitungselektronen, Z. Phys. 80, 763 (1933).

[38] S. Ejima, T. Kaneko, F. Lange, S. Yunoki, and H. Fehske, Photoinduced $\eta$-pairing in one-dimensional Mott insulators, JPS Conf. Proc. 30, 011184 (2020).

[39] S. Ejima, T. Kaneko, F. Lange, S. Yunoki, and H. Fehske, Photoinduced $\eta$-pairing at finite temperatures, Phys. Rev. Res. 2, 032008(R) (2020).

[40] P. T. Brown, E. Guardado-Sanchez, B. M. Spar, E. W. Huang, T. P. Devereaux, and W. S. Bakr, Angle-resolved photoemission spectroscopy of a Fermi-Hubbard system, Nat. Phys. 16, 26 (2019).

[41] R. G. Pereira, K. Penc, S. R. White, P. D. Sacramento, and J. M. P. Carmelo, Charge dynamics in half-filled Hubbard chains with finite on-site interaction, Phys. Rev. B 85, 165132 (2012).

[42] S. Ejima, H. Fehske, and F. Gebhard, Dynamic properties of the one-dimensional Bose-Hubbard model, Europhys. Lett. 93, 30002 (2011).

[43] J. K. Freericks, H. R. Krishnamurthy, M. A. Sentef, and T. P. Devereaux, Gauge invariance in the theoretical description of time-resolved angle-resolved pump/probe photoemission spectroscopy, Phys. Scr. 2015, 014012 (2015).

[44] Y. Wakisaka, T. Sudayama, K. Takubo, T. Mizokawa, M. Arita, H. Namatame, M. Taniguchi, N. Katayama, M. Nohara, and H. Takagi, Excitonic Insulator State in $\mathrm{Ta}_{2} \mathrm{NiSe}_{5}$ Probed by Photoemission Spectroscopy, Phys. Rev. Lett. 103, 026402 (2009).

[45] Y. Wakisaka, T. Sudayama, K. Takubo, T. Mizokawa, N. L. Saini, M. Arita, H. Namatame, M. Taniguchi, N. Katayama, M. Nohara, and H. Takagi, Photoemission spectroscopy of $\mathrm{Ta}_{2} \mathrm{NiSe}_{5}$, J. Supercond. Nov. Magn. 25, 1231 (2012).

[46] M. Fishman, S. R. White, and E. M. Stoudenmire, The ITensor software library for tensor network calculations, arXiv:2007.14822.

[47] F. H. L. Essler, H. Frahm, F. Göhmann, A. Klümper, and V. E. Korepin, The One-Dimensional Hubbard Model (Cambridge University Press, Cambridge, 2005).

[48] H. N. Phien, G. Vidal, and I. P. McCulloch, Infinite boundary conditions for matrix product state calculations, Phys. Rev. B 86, 245107 (2012). 